\title{
The Path to Quantitative 3D Metrology: FIB-SEM 3D Tomography in Data Storage Industry
}

\author{
Zhi-Peng $\mathrm{Li}^{1}$, Jianxin Fang ${ }^{1}$ and Haifeng Wang ${ }^{1}$ \\ 1. Materials Science Lab, Western Digital Corporation, Fremont, USA.
}

Technology evolution in data storage industry is not only driven by the continuous scaling down of devices, but also by an increasing complexity of the critical elements in the devices, e.g., sensors, magnetic head in data storage industry. Physical characterization of these elements is required to support advanced product design and process development to produce such devices. However, for three dimensional and geometry sensitive features, conventional 2D techniques may only provide partial results that are less relevant or misleading due to the limitation of the information extracted from the feature in arbitrary perspective or in projection. The third dimension information is accessible by 3D imaging. Therefore, 3D quantitative analysis becomes increasingly important and essential in providing holistic and accurate information [1-3]. Focused ion beam (FIB) - scanning electron microscope (SEM) tomography can enable characterization a device over its entire volume, while maintaining the resolution of SEM. In this study, we will introduce the quantitative 3D tomography technique developed in the data storage industry to specifically address the industrial process development needs. In details, the methodology of 3D reconstruction will be introduced, key parameters will be reviewed, and the steps for a successful 3D tomography will be described. Typical applications of 3D analysis for device design and process development are presented to illustrate the benefits and unique learning opportunities.

A 3D tomography characterization relies on two distinct sections. Firstly, it needs to slice and image the device. Fig. 1a shows the dual-beam system used in this study, an integrated FIB-SEM equipment. FIB was initially applied to mill the sample. As long as the cross-sectional region of interest was exposed, the SEM image was acquired and saved accordingly. Normally, there is a $45^{\circ}$ between the FIB and SEM guns. In the subsequent steps more materials will be sputtered and cleaned by FIB (step width can be tuned by recipe), followed by SEM imaging. The desired region of the device will be milled by FIB into multiple slices. Following each milling sequence, the SEM image was acquired immediately. Once the entire region of interest has been completely sliced and images, the recorded SEM images are stacked into a dataset, as shown in Fig. 1b. In order to obtain quantitative 3D tomography data, the slice intervals should be recorded each time when one FIB mill was accomplished. This numerical data of slice step is crucial for the following 3D reconstruction in order to obtain quantitative 3D tomography result. With this aim, FIB needs to be maintained in a reliable and high quality condition in order to fulfill the whole cutting and imaging processes, which usually took several hours. Note that for hours of sectioning devices by FIB, it is difficult to control even slice interval, especially by manual operation. Therefore, an in-house auto-recipe was developed to automatically control FIB operation, which can enable FIB operated by similar condition during hours of working time. Fig. 1c displays the slice intervals measurement for hundreds of images, which clearly represents the linear relationship among all recorded images as well as even slice interval.

After collecting all the sliced images, the image dataset is then aligned and filtered to reconstruct the whole volume of the device. It needs to emphasize that the alignment of all images are extremely critical, in order to obtain accurate 3D structure without distortion or artefact. Fig. 1c clearly demonstrates the well controlled FIB cutting, enabling the high quality 3D reconstruction. Moreover, by 
tuning the FIB beam condition, the FIB cutting step size can be tuned accordingly, from several nanometers to $\sim 50$ nanometers. The step width depends on the resolution that is required for a given device. The whole volume of milled area in the device can also be tuned by auto-recipe from a very small region (i.e., several nanometers ${ }^{3}$, Fig. 2a), to a chunk of materials as large as several micrometers ${ }^{3}$ (Fig. 2b).

References:

[1] K. Lepinay and F. Lorut, Microsc. Microanal. 19, (2013), p. 85.

[2] P. G. Kotula, M. R. Keenan and J. R. Michael, Microsc. Microanal. 12 (2006), p. 36.

[3] G. Mobus, R. C. Doole, B. J. Inkson, Ultramicroscopy 96 (2003), p. 433.

(a)

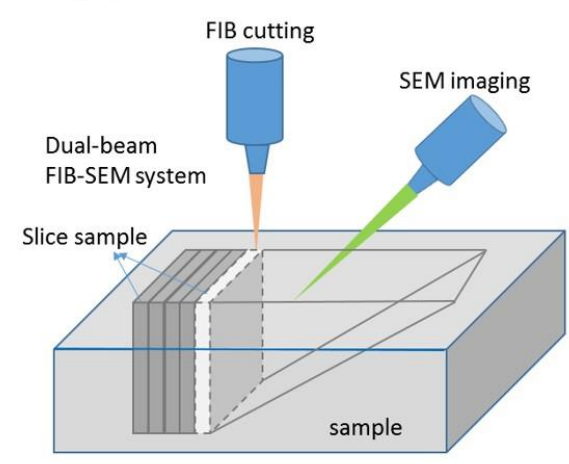

(b)

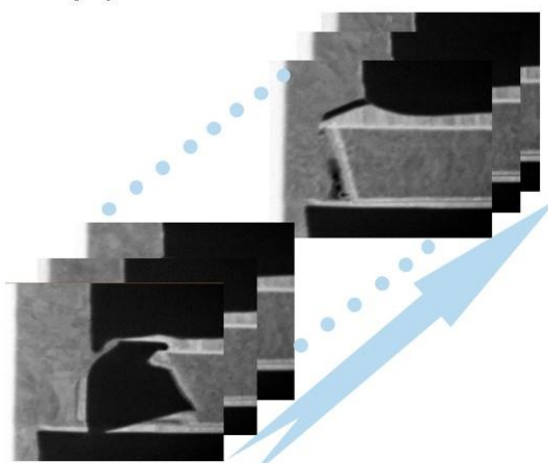

(c)

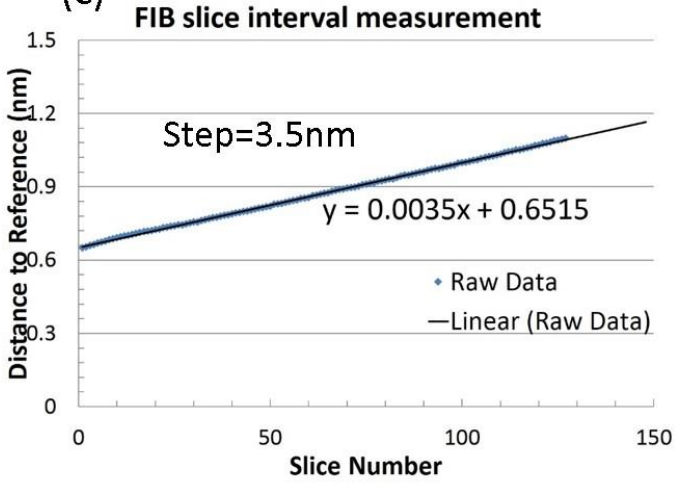

Figure 1. (a) Schematic diagram of dual-beam system used in this study. (b) Series of cross-sectional SEM images of samples by FIB slicing. (3) Slice intervals of each images shown in (b).

(a) $0.5 \times 0.5 \times 0.5 \mu m^{3}$

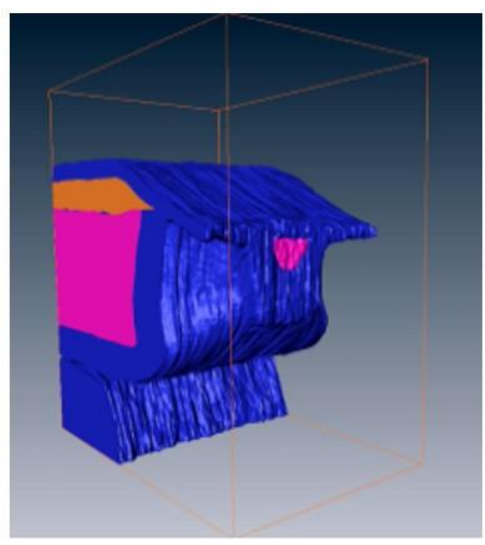

(b) $5 \times 5 \times 5 \mu \mathrm{m}^{3}$

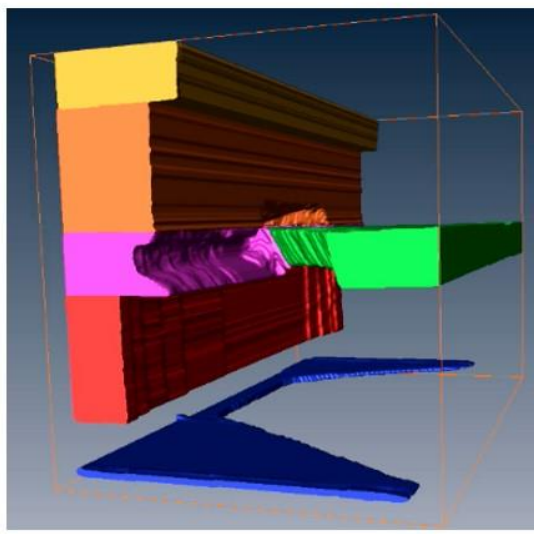

Figure 2. Reconstructed 3D images of (a) a small region of device $\left(0.5 \mu \mathrm{m}^{3}\right)$, (b) a large area of device $\left(5 \mu \mathrm{m}^{3}\right)$. 